\title{
Urban farming, una soluzione per lo sviluppo sostenibile delle città
}

\author{
Maria Federica Siani ${ }^{1}$ \\ ${ }^{1}$ Università degli Studi di Salerno
}

\begin{abstract}
L'agricoltura urbana può giocare un ruolo fondamentale nello sviluppo sostenibile delle città poiché permette non solo di soddisfare la domanda crescente di cibo riducendo le criticità dell'attuale filiera alimentare, ma anche di apportare diversi benefici dal punto di vista ambientale, sociale ed economico.

Lo studio evidenzia le principali tipologie di quelle che vengono definite urban farms ed i sistemi necessari per realizzarle, riportando a supporto un'applicazione nell'ambito di un progetto di recupero di un ex stabilimento industriale nei pressi del Parco Archeologico di Paestum (SA).

I risultati mostrano come l'integrazione dell'agricoltura, ed eventualmente dell'allevamento, possa essere utile per il recupero degli spazi inutilizzati nelle città rendendoli parte attiva dei processi che si consumano al loro interno. Queste pratiche permettono di portare i processi naturali nell'ambiente urbano, riducendo allo stesso tempo alcuni dei fattori critici ambientali e incentivando il benessere sociale e la creazione di nuove economie.
\end{abstract}

\section{Le soluzioni integrate e le tecniche innovative di agricoltura ur-}

\section{bana}

La crescente urbanizzazione e i cambiamenti climatici in atto pongono il problema dell'approvvigionamento delle risorse al centro del dibattito sullo sviluppo sostenibile delle 
città $^{1,2}$.

L'agricoltura urbana offre una preziosa opportunità poiché, accorciando la filiera alimentare, permette di ridurre i fattori critici legati ai trasporti su lunga distanza, come le emissioni di gas serra e gli sprechi alimentari, e di fornire prodotti di alta qualità, freschi e locali agli abitanti, coinvolgendoli sia dal punto di vista educativo che lavorativo ${ }^{1,3}$.

Negli ultimi anni si stanno diffondendo i progetti definiti di “Zero-Acreage Farming”, ovvero realizzati sulle coperture o all'interno di edifici esistenti e caratterizzati dal non-utilizzo di superficie 1. Si distinguono principalmente in:

- orti pensili, sistemi intensivi a verde pensile destinati alla coltivazione di frutta e ortaggi ${ }^{4}$;

- serre su tetto;

- "vertical farms" o "plant factories" o “indoor farms", che utilizzano sistemi multipiano verticali per la produzione di cibo all'interno di edifici nuovi o già esistenti ${ }^{4,5,6}$.

Le serre su tetto e le vertical farms, diversamente dagli orti pensili che si basano su metodi di produzione meno sofisticati basati sul suolo, utilizzano tecniche di coltura fuori suolo abbinate in alcuni casi a pratiche di allevamento ${ }^{7,6,1}$.

La coltivazione di piante senza l'uso di terreno, definita idroponica, comprende sia i sistemi che includono un substrato inerte, "substrate-based hydroponics" o "media bed technique”, sia i sistemi che utilizzano solo soluzione nutritiva e aria senza l'utilizzo, o quasi, di un substrato solido ${ }^{8,9}$. Tra questi ultimi rientrano ${ }^{8,10}$ :

- la “Deep Flow Technique” o “Deep Water Culture” (DWC), in cui le piante sono sospese su un supporto galleggiante con le radici immerse in una vasca contenente la soluzione nutritiva;

- la "Nutrient Film Technique" (NFT), in cui le piante crescono in fori praticati in canali orizzontali con le radici che ricevono un rivolo continuo di soluzione nutritiva (Fig. 1);

- l'aeroponica, dove le piante sono supportate da pannelli e la soluzione nutritiva viene nebu- 
lizzata direttamente sulle radici sospese ad intervalli regolari.

I sistemi idroponici possono essere a ciclo chiuso o aperto, a seconda che si preveda o meno il ricircolo della soluzione nutritiva, e, nel caso di utilizzo di substrato, quest'ultimo può essere riutilizzato in seguito a sterilizzazione ${ }^{8,10}$.

L'integrazione dell'idroponica con l'acquacoltura a ricircolo, cui ci si riferisce con l'acronimo RAS ("Recirculating Aquaculture Systems"), ovvero l'allevamento di pesci in vasche con il ricircolo dell' acqua in seguito ad un doppio processo di filtrazione meccanica e biologica, viene definita acquaponica, un sistema di produzione in cui le acque di scarto dell'acquacoltura vengono utilizzate per fornire nutrienti alle piante coltivate con uno dei sistemi idroponici sopra descritti ${ }^{8,12}$. In particolare, in seguito alla rimozione dei rifiuti solidi e alla conversione dell'ammoniaca nel processo di nitrificazione, i nitrati e le altre sostanze nutritive disciolte nell'acqua vengono assorbite dalle radici delle piante e l'acqua drenata ritorna purificata alle vasche dei pesci ${ }^{8}$.

Si rileva che le emissioni odorigene nei sistemi acquaponici rappresentano una problematica che va trattata ed analizzata secondo le migliori tecnologie all' avanguardia che oggi la ricerca può mettere in campo ${ }^{13,14}$.

La Figura 2 che segue mostra il funzionamento di un sistema acquaponico a ciclo unico.

I sistemi acquaponici rappresentano ad oggi una buona tecnica volta a rigenerare i progetti urbani e potrebbe essere anche una buona pratica in grado di rimuovere i contaminanti emergenti dalle acque reflue ${ }^{16,17,18,19}$. Pertanto sono stati condotti alcuni studi sui sistemi acquaponici in cui viene evidenziata la funzionalità del processo e la sua fattibilità tecnica ed economica ${ }^{20,21,22,23}$.

Le tecniche innovative di urban farming permettono, oltre alla produzione in qualsiasi tipo di ambiente, il controllo e la riduzione di malattie e parassiti terricoli, una maggiore qualità e quantità delle colture e un minore consumo di acqua, fertilizzanti e terreno ${ }^{8}$. Instaurando, poi, delle sinergie con gli edifici ospitanti in termini di scambio delle risorse, quali acqua, aria ed energia, è possibile 
realizzare quella che Gould e Caplow definiscono “Building-Integrated Agriculture”, che permette la produzione di cibo a km 0 per gli abitanti e il contemporaneo miglioramento delle prestazioni energetiche degli edifici e, di conseguenza, della qualità ambientale dell'intorno ${ }^{24,1}$.

\section{La sostenibilità delle pratiche di urban farming}

Praticare l'agricoltura e l'allevamento sulle coperture degli edifici e al loro interno comporta numerosi benefici sia diretti che indiretti, i primi sono quelli direttamente legati all'integrazione delle soluzioni di urban farming nelle strutture ospitanti, i benefici indiretti sono, invece, quelli che derivano dall'accorciamento della filiera alimentare e dall'utilizzo di tecniche più efficienti rispetto a quelle dell'agricoltura tradizionale ${ }^{7,25}$.

Le sfide maggiori per l'attuazione di queste pratiche sono quelle legate agli aspetti strutturali e normativi e ai progressi tecnologici ancora necessari in campo energetico, ma il riutilizzo delle risorse che altrimenti andrebbero perse nei processi urbani può assicurarne la sostenibilità sotto tutti gli aspetti ${ }^{1,25,5}$.

Una preziosa opportunità è ad esempio offerta dal recupero delle acque piovane, delle acque reflue e dei rifiuti organici che possono essere utilizzati per fornire acqua, energia e nutrienti, inputs fondamentali per la produzione di cibo, andando allo stesso tempo ad alleggerire il carico sui rispettivi sistemi di drenaggio e di trattamento ${ }^{7,25}$.

Nel capitolo successivo verrà presentato un caso studio relativo ad un ex stabilimento industriale nei pressi del Parco Archeologico di Paestum per il quale si prospetta l'integrazione di sistemi di agricoltura urbana nell' ambito di un progetto di recupero. 


\section{Un'applicazione progettuale: urban farming nell'ex fabbrica Ci-}

\section{rio a Paestum}

L'ex fabbrica Cirio situata in località Santa Venera, a sud di Paestum (SA), è un complesso industriale degli inizi del '900 rimasto attivo fino alla fine degli anni '70 per la lavorazione dei pomodori ed oggi si presenta in evidente stato di abbandono e degrado, nonostante sia un esempio di archeologia industriale ed inglobi parte dei resti di un bene archeologico di interesse culturale dichiarato risalente al V secolo a.C., il santuario di Santa Venera ${ }^{26,27}$.

Il sito, che rientra nella competenza territoriale del Parco Archeologico di Paestum e Velia, è stato scelto in quanto inserito in un territorio che attrae centinaia di migliaia di visitatori ogni anno e in continuo sviluppo e quindi si può adattare bene ad una rifunzionalizzazione che lo renda parte attiva dell'offerta della zona ${ }^{26}$.

Nelle Figure 3 e 4 seguenti si riportano le piante del piano terra e del primo piano rappresentative dello stato di fatto. L'ex fabbrica Cirio è composta da 5 capannoni e 3 edifici sul lato strada e il capannone $\mathrm{H}$ più decentrato e il grande piazzale interno sono stati individuati per l'integrazione di pratiche di urban farming.

La scelta di ricollegarsi alla storia del sito prevedendo la coltivazione di pomodori in loco con una delle tecniche innovative di urban farming è stata naturale.

Sulla base degli spazi a disposizione si è previsto di installare un sistema acquaponico del tipo "decoupled" su due livelli nel capannone $\mathrm{H}$, di superficie coperta pari a $521 \mathrm{~m}^{2}$ e altezza interna netta di 6 m. I sistemi disaccoppiati, i "Double Recirculation Aquaponic Systems", sono costituiti da due circuiti indipendenti, quello dell' acquacoltura a ricircolo e quello dell'idroponica, 
che operano insieme, così da ottimizzare i valori di $\mathrm{pH}$, temperatura e concentrazione dei nutrienti per ciascuno di essi ${ }^{12}$.

È stato, dunque, previsto il posizionamento del circuito RAS al piano terra in un locale di $481 \mathrm{~m}^{2}$ e del circuito idroponico in una serra al primo piano, della stessa superficie e con un'altezza di gronda di $3 \mathrm{~m}$, rispettando la volumetria attuale.

Per il circuito idroponico si è scelto di utilizzare la tecnica dei media beds in un sistema a ciclo chiuso sopraelevato costituito da sacche di substrato organico, posizionate lungo canali, nelle quali vengono trapiantate le giovani piante fatte propagare nello stesso tipo di substrato ma in forma pressata $^{28}$. La fertirrigazione avviene tramite il "drip system" tipo "microtube”, ovvero con più microtubi di alimentazione da cui si dipartono dei gocciolatori, ciascuno dei quali serve una pianta ad intervalli regolari, e l'acqua drenata viene raccolta e fatta ricircolare ${ }^{28}$.

Si è progettato dunque di disporre 17 sacche di crescita, ciascuna della lunghezza di $120 \mathrm{~cm} \mathrm{e}$ contenente 3 piante, su canali in 12 file, per un totale di 612 piante. Data la produttività riportata per sistemi idroponici simili a quello descritto, il raccolto annuo previsto è di $6.120 \mathrm{~kg}$ di pomodori ${ }^{29}$. Noto il numero delle piante, è stato effettuato un dimensionamento di massima del circuito RAS secondo il metodo del "feed rate ratio", che consente di bilanciare un sistema acquaponico sulla base del rapporto tra la quantità giornaliera di mangime per pesci immessa e la superficie di coltivazione delle piante ${ }^{8}$. Effettuando una similitudine con la coltivazione in cassoni, sono stati così calcolati la quantità di mangime, il numero di pesci ed il volume di acqua totale delle vasche. In particolare, sono state previste 10 vasche da $17 \mathrm{~m}^{3}$ ciascuna per allevare $1.632 \mathrm{~kg}$ di persico trota (Micropterus salmoides), pesce d'acqua dolce che si adatta bene all'allevamento nei sistemi acquaponici nelle regioni con clima mediterraneo e ha ottimi valori nutrizionali e qualità culinarie ${ }^{8}$.

Per la filtrazione meccanica si è previsto un filtro automatico a tamburo, un tipo di “microscreen” che separa i solidi di dimensioni superiori a 40-100 $\mu \mathrm{m}$ grazie ad un elemento filtrante 
e, all'attivazione di sensori del livello dell'acqua, ruotando li indirizza al canale di raccolta dei fanghi e quindi al sistema di trattamento esterno ${ }^{30,15,31}$. Per la biofiltrazione si è previsto un "Moving Bed Biofilm Reactor" (MBBR), un sistema dotato di supporti di plastica liberi di muoversi in acqua e con un'elevata area superficiale che permette lo sviluppo di colonie di batteri nitrificanti, responsabili della conversione dell' ammoniaca $\left(\mathrm{NH}_{3}\right)$ prima in nitriti $\left(\mathrm{NO}_{2}{ }^{-}\right)$e poi in nitrati $\left(\mathrm{NO}_{3}{ }^{-}\right)^{8,30,32}$. Entrambi i sistemi di filtrazione sono stati dimensionati sulla base dei dati suggeriti in letteratura.

Poiché il sistema progettato è a bassa densità di stoccaggio, si può prevedere un ricircolo dell'acqua ogni ora ${ }^{8}$.

Considerando un consumo giornaliero dell' $1 \%$ del volume totale di acqua delle vasche, per il reintegro si è previsto l'utilizzo di acqua piovana raccolta dalle coperture della serra e di parte degli edifici del complesso ${ }^{8}$. L'acqua piovana è una delle migliori fonti per un sistema acquaponico in quanto generalmente, oltre ad essere molto pulita, è caratterizzata da ${ }^{8,28}$ :

- $\mathrm{pH}$ neutro;

- bassi valori di durezza;

- salinità quasi nulla;

- assenza di cloro;

- assenza di tossine e agenti patogeni.

Si è prevista, dunque, l'installazione di un “pumped system”, in cui l'acqua dai pluviali, in seguito alla rimozione di eventuali detriti tramite un filtro autopulente, viene immagazzinata in un serbatoio fuori terra o interrato da cui viene poi pompata per i diversi utilizzi ${ }^{33}$.

Il dimensionamento del serbatoio è stato effettuato secondo il metodo semplificato proposto dalla norma UNI/TS 11445:2012, che ne valuta il volume utile sulla base dell'afflusso meteorico annuo e della domanda idrica annua ${ }^{34}$. Il volume ottimale del serbatoio destinato a rifornire il sistema 
acquaponico recuperando le acque piovane dalle coperture della serra e degli edifici $\mathrm{D}$, E, I è pari a $60 \mathrm{~m}^{3}$.

Si è previsto, infine, di collegare lo scarico di troppo pieno del serbatoio interrato a pozzi perdenti, un sistema sotterraneo di infiltrazione che rientra tra quelli che vengono definiti "soakaways" 33 . I pozzi perdenti sono costituiti da anelli forati prefabbricati sovrapposti l'uno sull'altro che consentono di immagazzinare temporaneamente le acque piovane, che poi si infiltrano in maniera concentrata nel sottosuolo ${ }^{33}$.

Per il dimensionamento dei pozzi perdenti si fa riferimento alla norma tedesca DWA-A 138, che ne permette di calcolare l'altezza utile sulla base dei dati relativi a:

- precipitazioni massime riferite ad una data durata;

- superficie di raccolta dell'acqua;

- tipo di terreno drenante;

- caratteristiche dell'anello perdente.

Per il caso in esame risulta che sarebbero necessari 3 punti pozzo con diametro di 2,65 m e profondità totale di 3,50 m.

In Figura 5 è riportata la schematizzazione del funzionamento del sistema acquaponico progettato.

Nel piazzale interno del complesso è stata prevista l'installazione di 145 “torri” per la coltivazione idroponica verticale.

Questi sistemi sono costituiti da torri in plastica per alimenti stabilizzata ai raggi UV, ciascuna alta 2,46 m e con una riserva alla base di circa 761 che permette di produrre con la tecnologia 
aeroponica fino a 44 piante $^{35}$. La produttività mensile riportata è di 170 grammi per pianta ed è necessario predisporre un serbatoio a gravità e un sistema di dosaggio dei nutrienti e/o del pH per l'apporto della soluzione nutritiva a ciascuna torre ${ }^{35}$.

Si è previsto, dunque, di coltivare verdure a foglia ed erbe aromatiche di stagione, per un raccolto totale annuo di circa $13.000 \mathrm{~kg}$.

Installando una pavimentazione permeabile in parte del piazzale interno è possibile recuperare le acque piovane per rifornire le torri per la coltivazione idroponica verticale. Il sistema di pavimentazione permeabile senza infiltrazione può essere, infatti, collegato ad una struttura geocellulare che funge da serbatoio ${ }^{33}$.

Per il dimensionamento di massima del volume della struttura geocellulare necessario a soddisfare il fabbisogno idrico si è fatto riferimento alla norma UNI/TS 11445:2012 di cui sopra, considerando i seguenti dati:

- il consumo giornaliero di acqua riportato per una torre aeroponica è di circa $3,801^{35}$;

- la pavimentazione permeabile totale che si prevede di installare è di $618 \mathrm{~m}^{2}$.

Il volume ottimale del sistema geocellulare risulta pari a $20 \mathrm{~m}^{3}$.

Partendo dalle attività di urban farming previste per l'ex stabilimento Cirio, sono state definite le nuove destinazioni d'uso per i restanti capannoni e edifici costituenti il complesso nell'ottica della realizzazione di una nuova porta di accesso al Parco Archeologico di Paestum.

In particolare sono stati previsti:

- depositi archeologici visitabili nel capannone A;

- sala mostra dedicata alla dieta mediterranea nel capannone G; 
- ristorante con lounge bar nel capannone B;

- sala conferenze nel capannone C;

- guardiania e uffici nell'edificio D;

- servizi di accoglienza e uffici nell'edificio E;

- negozio per la vendita al dettaglio dei prodotti, ingresso e spogliatoi per il personale e locale tecnico nell'edificio I.

Nella zona esterna a est del complesso si è prevista la realizzazione di un sistema di orti comunitari in affitto per la coltivazione tradizionale su suolo di prodotti destinati al consumo personale e all'economia sociale e solidale, che permetta anche lo sviluppo di iniziative di inclusione, formazione e inserimento lavorativo di soggetti svantaggiati.

\section{Conclusioni}

Le soluzioni integrate di urban farming sono, dunque, efficacemente applicabili in qualsiasi contesto urbano, previa verifica di compatibilità strutturale nel caso di edifici esistenti. Tale tipo di integrazione ha risvolti positivi sotto tutti gli aspetti della sostenibilità: ambientale, sociale ed economico.

Il progresso tecnologico, in particolare nel campo energetico e della produzione di materiali più sostenibili a lungo termine, potrà rendere sempre più accessibili questo tipo di soluzioni, ma è necessario uno sforzo costante da parte delle istituzioni per incentivarle e finanziarle.

Lo sviluppo sostenibile delle città sarà un obiettivo da perseguire con sempre maggiore tenacia nei prossimi anni e le soluzioni integrate di urban farming, coprendo gli aspetti sia architettonici, che urbanistici, che di gestione delle risorse, offrono una preziosa opportunità per il suo raggiungimento e mantenimento. 


\section{References}

1.Thomaier, S. et al.. Farming in and on urban buildings: Present practice and specific novelties of Zero-Acreage Farming (ZFarming). Renewable Agriculture and Food Systems 30, 43-54 (2014).

2.FAO. Urban Food Agenda — Food and Agriculture Organization of the United Nations. (2020).

3.Weidner, T. \& Yang, A. The potential of urban agriculture in combination with organic waste valorization: Assessment of resource flows and emissions for two european cities. Journal of Cleaner Production 244, 118490 (2020).

4.Al-Kodmany, K. The Vertical Farm: A Review of Developments and Implications for the Vertical City. Buildings 8, 24 (2018).

5.Despommier, D. Vertical farms, building a viable indoor farming model for cities. Field Actions Science Report 2019, 68-73 (2019).

6.Goodman, W. \& Minner, J. Will the urban agricultural revolution be vertical and soilless? A case study of controlled environment agriculture in New York City. Land Use Policy 83, 160-173 (2019).

7.Ackerman, K. Urban agriculture: opportunities and constraints. in Metropolitan Sustainability 118-146 (Elsevier, 2012). doi:10.1533/9780857096463.2.118.

8.Somerville, C., Cohen, M., Pantanella, E., Stankus, A. \& Lovatelli, A. Small-scale aquaponic food production. Integrated fish and plant farming. 262 (FAO, 2014).

9.Raviv, M., Lieth, J. H. \& Bar-Tal, A. Significance of Soilless Culture in Agriculture. in Soilless Culture 3-14 (Elsevier, 2019). doi:10.1016/b978-0-444-63696-6.00001-3.

10.Maucieri, C. et al.. Hydroponic Technologies. in Aquaponics Food Production Systems 77-110 (Springer International Publishing, 2019). doi:10.1007/978-3-030-15943-64. 
11.Gotham Greens. Gotham Greens. (2020).

12.Goddek, S., Joyce, A., Kotzen, B. \& Dos-Santos, M. Aquaponics and Global Food Challenges. in Aquaponics Food Production Systems 3-17 (Springer International Publishing, 2019). doi:10.1007/978-3-030-15943-6 ${ }_{1}$.

13.Zarra, T., Naddeo, V. \& Belgiorno, V. A novel tool for estimating the odour emissions of composting plants in air pollution management. Issue 4 11, 477-486 (2013).

14.Naddeo, V., Zarra, T., Giuliani, S. \& Belgiorno, V. Odour Impact Assessment in Industrial Areas. Chemical Engineering Transactions (CET) 30, (2012).

15.Palm, H. W., Knaus, U., Appelbaum, S., Strauch, S. M. \& Kotzen, B. Coupled Aquaponics Systems. in Aquaponics Food Production Systems 163-199 (Springer International Publishing, 2019). doi:10.1007/978-3-030-15943-6 .

16.Scannapieco, D., Naddeo, V. \& Belgiorno, V. Sustainable power plants: A support tool for the analysis of alternatives. Land Use Policy 36, 478-484 (2014).

17.Nesticò, A., Elia, C. \& Naddeo, V. Sustainability of urban regeneration projects: Novel selection model based on analytic network process and zero-one goal programming. Land Use Policy 99, 104831 (2020).

18.Naddeo, V. Development of environmental biotechnology and control of emerging biological contaminants: the grand challenge for a sustainable future. Water Environment Research 92 , 1246-1248 (2020).

19.Naddeo, V., Belgiorno, V., Zarra, T. \& Scannapieco, D. Dynamic and embedded evaluation procedure for strategic environmental assessment. Land Use Policy 31, 605-612 (2013).

20.Lombardo, L. Gestione avanzata delle risorse naturali con i sistemi acquaponici: analisi tecnica ed economica. (2020) doi:10.22541/au.160322479.92556918/v1. 
21.Bovenzi, F. L'ingegneria sanitaria ambientale a supporto dell'agricoltura urbana per lo sviluppo di un'economia circolare. (2020) doi:10.22541/au.160340490.03649842/v1.

22.Muro, G. D. Verso una agricoltura urbana sostenibile: i sistemi acquaponici per il recupero e il riutilizzo delle acque. (2020) doi:10.22541/au.158525190.07859900.

23.Regno, M. D. Processi naturali per il recupero e il riutilizzo delle acque nei sistemi acquaponici. (2020) doi:10.22541/au.158525193.30158307.

24.Gould, D. \& Caplow, T. Building-integrated agriculture: a new approach to food production. in Metropolitan Sustainability 147-170 (Elsevier, 2012). doi:10.1533/9780857096463.2.147.

25.Kalantari, F., Tahir, O. M., Joni, R. A. \& Fatemi, E. Opportunities and Challenges in Sustainability of Vertical Farming: A Review. Journal of Landscape Ecology 11, 35-60 (2018).

26.Parco Archeologico di Paestum e Velia. Parco Paestum e Velia - Parco Archeologico di Paestum e Velia. (2020).

27.MiBact. Sito web del Ministero per i beni e le attività culturali e per il turismo. (2020).

28.Os, E. A. van, Gieling, T. H. \& Lieth, J. H. Technical Equipment in Soilless Production Systems. in Soilless Culture 587-635 (Elsevier, 2019). doi:10.1016/b978-0-444-63696-6.00013-x.

29.Sanjuan-Delmás, D. et al. Environmental assessment of an integrated rooftop greenhouse for food production in cities. Journal of Cleaner Production 177, 326-337 (2018).

30.Bregnballe, J. A Guide to Recirculation Aquaculture. 100 (FAO and EUROFISH International Organisation, 2015).

31.Hydrotech. Hydrotech microscreen filters: Drumfilters, Discfilters and Beltfilters. (2020).

32.Espinal, C. A. \& Matulić, D. Recirculating Aquaculture Technologies. in Aquaponics Food Production Systems 35-76 (Springer International Publishing, 2019). doi:10.1007/978-3-030-15943$6{ }_{3}$. 
33.Woods Ballard, B. et al.. The SuDS Manual. 964 (CIRIA, 2015).

34.Gnecco, I., Palla, A., Campisano, A. \& Modica, C. Criteria for the Optimal Sizing of Rainwater Harvesting Systems in Europe. in Frontiers in Civil Engineering 83-114 (BENTHAM SCIENCE PUBLISHERS, 2017). doi:10.2174/9781681084831117020005.

35.The Juice Plus Company. Tower Farms. (2020). 


\section{Figure Captions}

Figure 1. Coltivazione di basilico con sistema NFT in una serra su tetto a New York City ${ }^{11}$

Figure 2. Sistema acquaponico semplice tipo "one-loop" con (A) vasca dei pesci e aerazione, (B) filtro meccanico, (C) biofiltro, unit@x @ $\mathrm{x}$ tempboxaa@spacefactor $\Gamma 18 a \Gamma @$ spacefactoridroponicatipo $(D) D W C,($ E)mediabedtechniqueo $(F) N F T e(C$

Figure 3. Pianta piano terra, ex stabilimento Cirio, Capaccio Paestum (SA)

Figure 4. Pianta primo piano, ex stabilimento Cirio, Capaccio Paestum (SA)

Figure 5. Schematizzazione del funzionamento del sistema acquaponico progettato per l'ex stabilimento Cirio (Siani, 2020) 


\section{Figures}

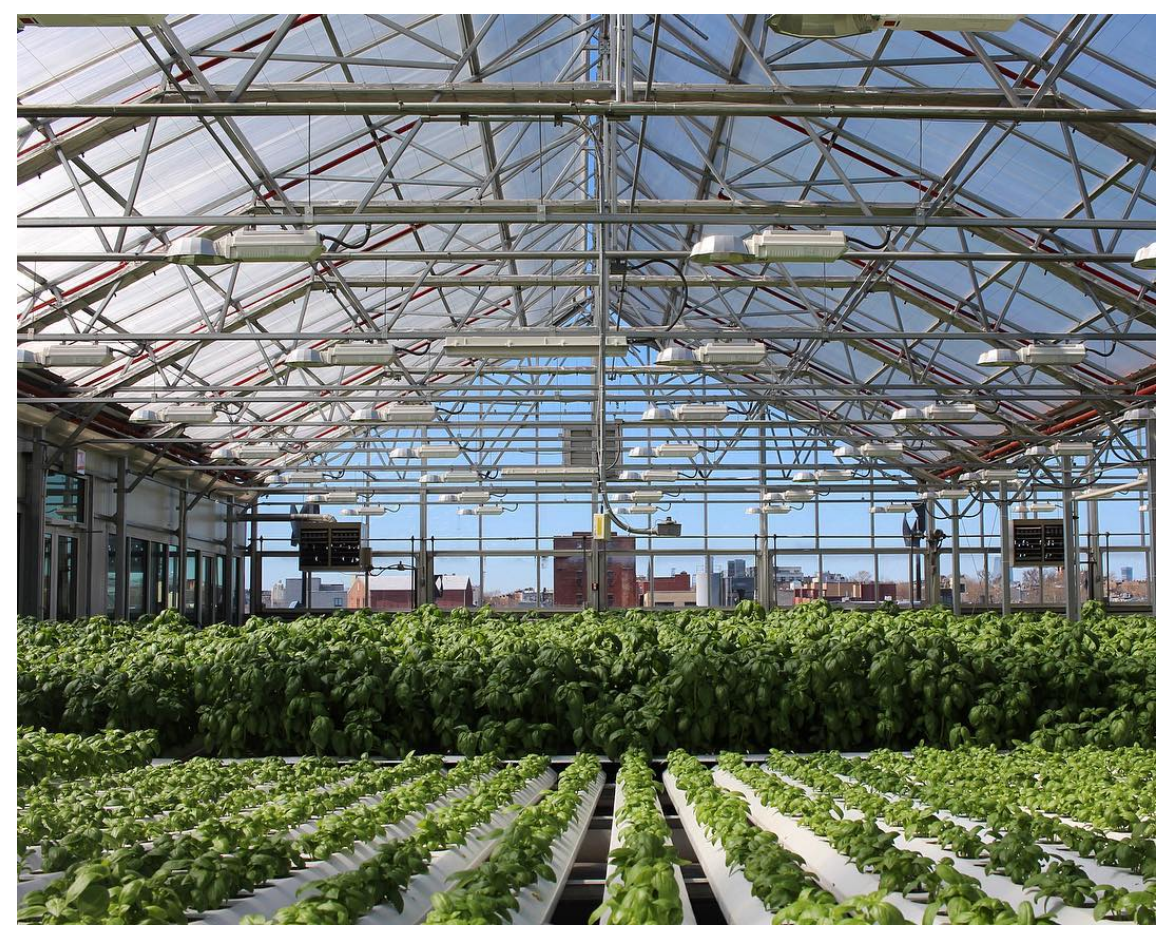

Figure 1: Coltivazione di basilico con sistema NFT in una serra su tetto a New York City ${ }^{11}$ 

(A)
(B)
(C) (D) (E) F

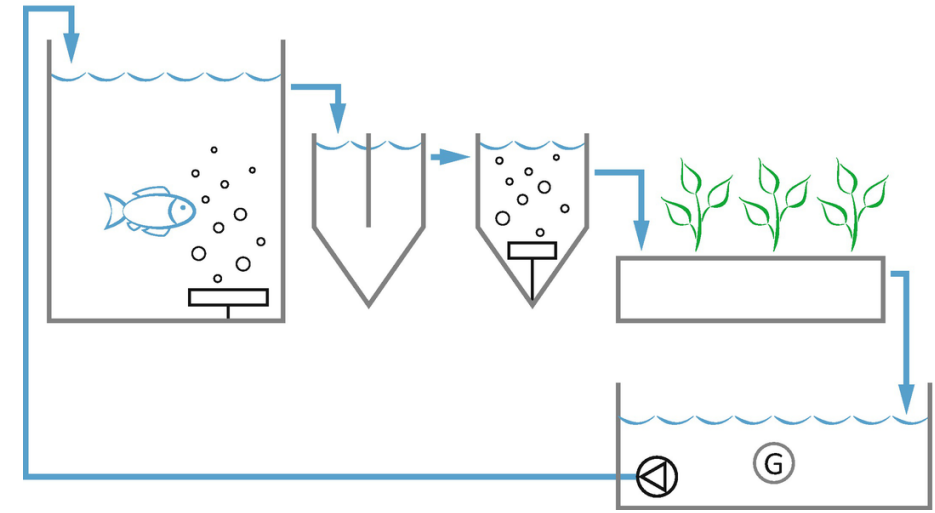

Figure 2: Sistema acquaponico semplice tipo "one-loop" con (A) vasca dei pesci e aerazione, (B) filtro meccanico, (C) biofiltro, unit@x @ $\mathrm{x}$ tempboxaa@spacefactor $\Gamma 18 a \Gamma @$ spacefactoridroponicatipo $(D) D W C,(E)$ mediabedtechniqueo $(F) N F T e(C$ 


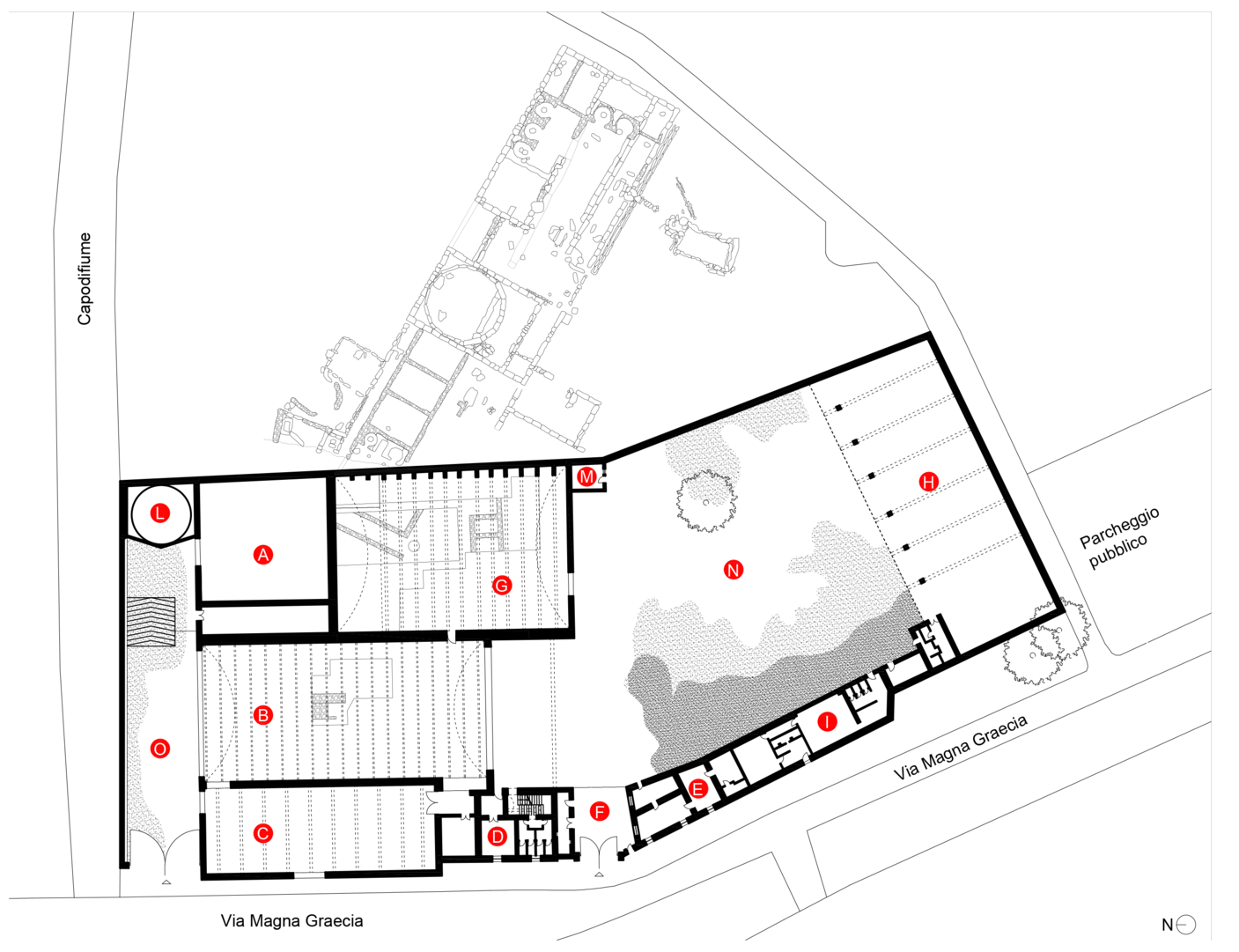

Figure 3: Pianta piano terra, ex stabilimento Cirio, Capaccio Paestum (SA) 


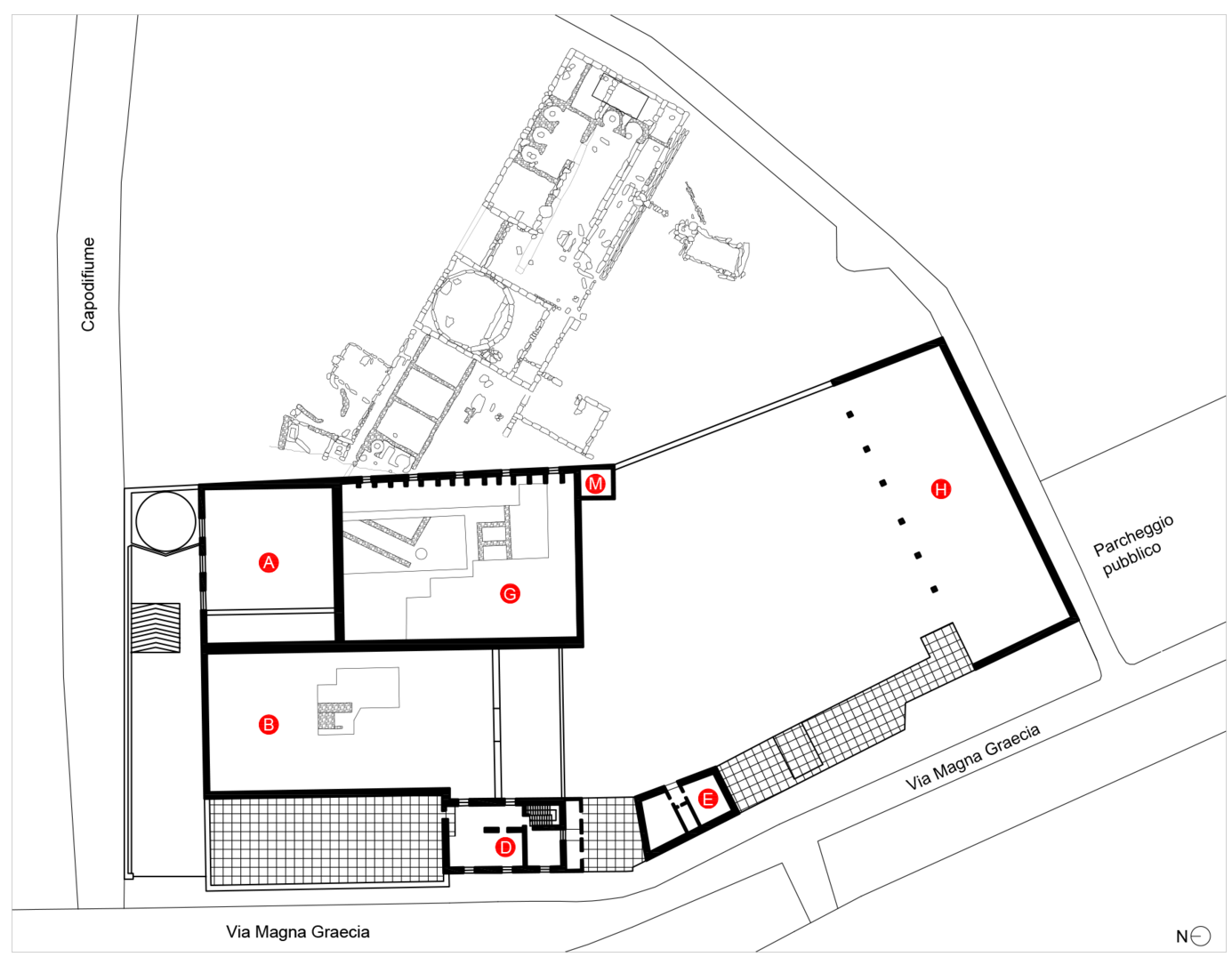

Figure 4: Pianta primo piano, ex stabilimento Cirio, Capaccio Paestum (SA) 


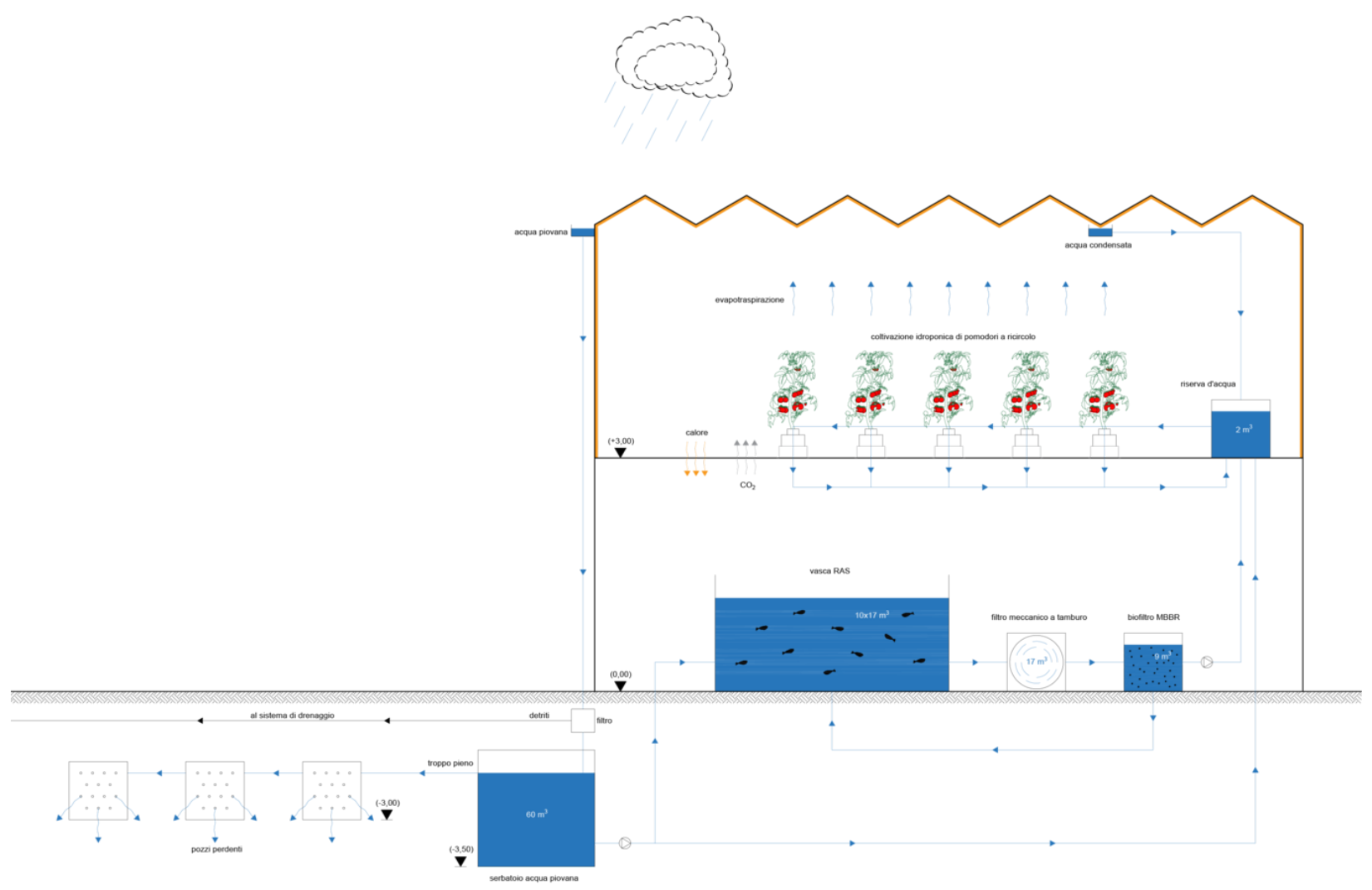

Figure 5: Schematizzazione del funzionamento del sistema acquaponico progettato per l'ex stabilimento Cirio (Siani, 2020) 